\title{
INVESTIGATION OF EARLY GROWTH OF CALCIUM HYDROXIDE CRYSTALS IN CEMENT SOLUTION BY SOFT X-RAY TRANSMISSION MICROSCOPY
}

\author{
V.S. Harutyunyan, ${ }^{1}$ A.P. Kirchheim, ${ }^{2}$ P.J.M. Monteiro ${ }^{2}$, A.P. Aivazyan, ${ }^{1}$ P. Fischer ${ }^{3}$ \\ ${ }^{1}$ Department of Solid State Physics, Yerevan State University, Yerevan, Armenia \\ ${ }^{2}$ Department of Civil and Environmental Engineering, University of California, Berkeley CA \\ ${ }^{3}$ Center for X-ray Optics, Lawrence Berkeley National Laboratory, Berkeley, CA USA
}

Research on cement hydration was performed at the full-field soft transmission X-ray microscope XM-1 located at beamline 6.1.2 at the Advanced Light Source (ALS) in Berkeley CA which is operated by the Center for X-ray Optics, Lawrence Berkeley National Laboratory, Berkeley, California. A series of works [1-3] has been conducted using this microscope for the insitu observation and qualitative analysis of through-solution hydration products and products of topochemical reactions, which form in cementitious aqueous solutions. This paper studies the precipitation of the calcium hydroxide $(\mathrm{CH})$ crystals from the cement solution. The analysis of successive images of the hydration process provides critical quantitative information about the growth rate of calcium hydroxide $(\mathrm{CH})$ crystals, the supersaturation ratio, and the kinetic and diffusion coefficients of the growth process.

ASTM Type II portland cement and $6 \% \quad \mathrm{C}_{4} \mathrm{~A}_{3} \overline{\mathrm{S}}$ admixture were mixed in aqueous solution and saturated with respect to $\mathrm{CH}$ and gypsum. The $\mathrm{C}_{4} \mathrm{~A}_{3} \overline{\mathrm{S}}$ admixture was included in the experimental program because of the general research program on expansive cements, and adding $\mathrm{C}_{4} \mathrm{~A}_{3} \overline{\mathrm{S}}$ to portland cement is an efficient method of generating ettringite and significant early-age expansion. The solution/solid materials ratio was $10 \mathrm{~cm}^{3} / \mathrm{g}$, which is higher than the one existing in regular concrete and mortars; to compensate for this dilution, the solution was originally saturated with $\mathrm{CH}$ and gypsum. To allow sufficient transmission of the soft X-rays, a small droplet was taken from the supernatant solution and assembled in the sample holder, and then squeezed between two silicon nitride windows for the analysis. The X-ray optical setup of the microscope XM-1 is described elsewhere [2].

In this experiment, a wavelength of $2.4 \mathrm{~nm}(516.6 \mathrm{eV})$ was used. The radiation transmitting the sample was detected using an X-ray CCD camera, with a resolution of $35 \mathrm{~nm}$ provided by Fresnel zone plate X-ray optics and magnification factor of about 2000. The recorded images have a circular field of view of approximately $10 \mu \mathrm{m}$ in diameter. The illumination time per image was in 
the range of 1 to 14 seconds depending largely on the absorption of the sample. The experimental work was conducted at room temperature $T=298 \mathrm{~K}$.

Figure 1 shows X-ray images of the hydration process during the first 118 minutes immediately after solution preparation procedure. The six images were recorded every 23.6 minutes, on average. The image in Fig. $1 a$ shows that during the first 15 minutes after the sample preparation, no formation of regularly-shaped crystals was observed. It is assumed that this timeinterval is associated with the nucleation period of the growing particles. The images presented in Figs. $1 b$ to $f$ clearly show the growth process of crystals with a plate-like shape. These crystals are imaged in different crystallographic projections that definitely belong to particles with a hexagonalprism shape, as is schematically depicted in Fig. 2. Meanwhile, as it is known from numerous experimental observations [4-7], the crystals of the $\mathrm{CH}$ precipitate with hexagonal-prism shape faceted by $\{10 \overline{10}\}$ prismatic and $\{0001\}$ basal facets and exhibit aspect ratio $r_{L}$ that satisfies the condition

$$
r_{L}=L_{1} / L_{2} \geq 1
$$

where $L_{1}$ and $L_{2}$ are the characteristic linear sizes of a crystal, corresponding to $<10 \overline{10}>$ and $<0001>$ growth directions as is shown in Fig. 2. Therefore, the regularly-shaped particles observed in our recorded images in Fig. 1 were assumed to be $\mathrm{CH}$ phase, but not ettringite. To a certain degree this assumption is supported by the X-ray image (see Fig. 3) recorded by us from aqueous solution saturated with respect to $\mathrm{CH}$ and gypsum and containing $100 \% \mathrm{C}_{4} \mathrm{~A}_{3} \overline{\mathrm{S}}$ admixture (i.e. no cement). On the one hand, in $100 \% \quad \mathrm{C}_{4} \mathrm{~A}_{3} \overline{\mathrm{S}}$ admixture-containing solution, most favorable conditions for formation exist for ettringite [8]. On the other hand, as a rule, in cemetitious materials ettringite precipitates as needle-like crystallites with hexagonal-prism shape and aspect ratio $r_{L}=L_{1} / L_{2}<1$ (Fig. 2 is valid also for depiction of growth facets of ettringite crystallites, for which, however, $L_{1}<L_{2}$ ). Clustering of precipitating crystallites is also typical to ettringite. Therefore, it is believed that the dense clusters of needle-like crystallites observed in image given in Fig. 3 belong to ettringite. Thus, it may be concluded that $6 \% \mathrm{C}_{4} \mathrm{~A}_{3} \overline{\mathrm{S}}$ admixture content in aqueous solution under investigation was not sufficient for promotion of the fast precipitation of ettringite particles with noticeable sizes during observation time.

As is clearly demonstrated in Figs. $1 \mathrm{~b}$ to $\mathrm{f}$, the relative locations and orientations of the growing $\mathrm{CH}$ crystals in solution are preserved in time, that is to say, during the growth process no strong convective fluxes were generated in solution and the growing particles remained immobile. 
For instance, in Figs. $1 \mathrm{~b}$ to $\mathrm{f}$, particle No.1 is viewed along a $<h k i 0>$ crystallographic direction, whereas particle No. 2 is oriented in such a way that its $\{0001\}$ basal facet (see also Fig. 2) is observed. Particle No. 1 is of special interest as it is viewed along a $<h k i 0>$ crystallographic direction (the $\{0001\}$ facets of the particle exhibit a slight mis-orientation with respect to observation direction), and its stable orientation was favorable for simultaneous measurements of the linear sizes $L_{1}$ and $L_{2}$ depending on time and, hence, for evaluation of corresponding growth rates in $<10 \overline{10}>$ and $<0001>$ growth directions (see Fig. 2).

The limitations of the microscope (a resolution of $35 \mathrm{~nm}$ ) does not allow observation of the nucleation process of the $\mathrm{CH}$ crystallites in solution from the very beginning of their formation. However, as a first approximation, it may be assumed that the nuclei of this phase possess a quasispherical shape and grow during the nucleation period, $0<t<t_{n}$, with a constant rate, $V_{n}$, that defines the increase of a nucleus diameter, $d$, with time according to a relationship [9]

$$
d(t)=V_{n} t
$$

where

$$
V_{n}=2 \Omega \beta_{n}\left(C-C_{e}\right)
$$

$t$ is the time, $t_{n}$ is the duration of the nucleation period, $\Omega$ is the volume occupied by a molecule (unit cell) in a nucleus, $\beta_{n}$ is the growth (nucleation) kinetic coefficient during the nucleation period, $C_{e}$ and $C$ (in $\mathrm{m}^{-3}$ units) are the equilibrium concentration (solubility) and actual concentration of solute molecules, respectively. At time $t=t_{n}$, only a small part of the $\mathrm{CH}$ crystallites reaches a critical diameter [9]

$$
d_{n}=\frac{4 \Omega \gamma}{k T \ln S}
$$

and continue to increase in size, whereas a majority of the $\mathrm{CH}$ crystallites with sizes in the range $d<d_{n}$ does not reach the critical diameter because of a partial dissolution that results from thermal and supersaturation fluctuations. In Eq. (4), $\gamma$ is the nucleus-water interfacial energy, $T$ is the temperature, $k$ is the Boltzmann constant, and $S$ is the supersaturation ratio of the solution. Equation (2) yields an alternative expression for the critical diameter, $d_{n}=V_{n} t_{n}$, which is achieved at $t=t_{n}$. The following simple relationships connect parameters $S, C_{e}$ and $C$ with the ionic product, $K$, and the solubility product, $K_{s p}$, of the $\mathrm{CH}$ : 


$$
S=\frac{K}{K_{s p}}, \quad C_{e}=\sqrt[3]{K_{s p} / 4}, \quad C=\sqrt[3]{K / 4}
$$

where parameters $C_{e} \approx 7.83 \times 10^{24} \mathrm{~m}^{-3}$ and $K_{s p} \approx 8.79 \times 10^{-6} \mathrm{M}^{3}$ are well established.

During the post-nucleation period, $t>t_{n}$, the $\mathrm{CH}$ nuclei that reached critical diameter continue to grow through their preferential $\{10 \overline{10}\}$ and $\{0001\}$ facets (see Fig. 2). For quantitative description of the growth process of $\mathrm{CH}$ particles at post-nucleation period, we may modify theoretical results obtained for growth process of a spherical particle in supersaturated solution (see e.g. $[9,10])$. The growth rate of a spherical particle, $V=\partial d / \partial t$, in a saturated and non-mixing solution in isotropic approximation is given by the following equation $[9,10]$ :

$$
\frac{\partial d}{\partial t}=\frac{2 \Omega \beta\left(C-C_{e}\right)}{1+\frac{\beta d}{2 D}}
$$

where $d$ is the diameter of particle, $\partial d / \partial t$ is the derivative with respect to time, $\beta$ is the isotropic growth kinetic coefficient that is assumed for a local surface area of the particle to be independent on crystallographic orientation, $D$ is the diffusion coefficient of solute particles, parameters $\Omega, C$ and $C_{e}$ have the same meaning as in Eq. (3). Note that in the limit of small sizes, $d \rightarrow 0$, Eq. (6) transforms into Eq. (3) once $\beta d /(2 D)<<1$. After integration of Eq. (6), one obtains

$$
d(t)=\sqrt{\left(\frac{2 D}{\beta}+d_{n}\right)^{2}+8 \Omega\left(C-C_{e}\right) D\left(t-t_{n}\right)}-\frac{2 D}{\beta},
$$

where it is implied that parameter $d_{n}=d\left(t_{n}\right)$ is the value of diameter at the beginning $t=t_{n}$ of the post-nucleation period, $t>t_{n}$, and is defined by Eq. (4). According to our experimental observations (see Figs. $1 b$ to $f$ ), the growing $\mathrm{CH}$ particles exhibit hexagonal-prism shape, and Eq. (7) is not valid for a realistic analysis of their shape evolution. To modify Eq. (7) for application to growth process of the $\mathrm{CH}$, it is assumed that the growth kinetic coefficient, $\beta$, and the equilibrium concentration of solute molecules, $C_{e}$, differ at $\{1010\}$ and $\{0001\}$ facets. This assumption is based on the following argumentation. The Curie-Wulff rule (see e.g. [9]) is valid at a size scale $1 \mu \mathrm{m}$ 
of a precipitating particle and for equilibrium configuration of a $\mathrm{CH}$ crystallite is expressed as follows:

$$
\frac{L_{1}}{L_{2}}=\frac{\gamma_{1}}{\gamma_{2}},
$$

where $\gamma_{1}$ and $\gamma_{2}$ are the interfacial energies between $\{10 \overline{10}\}$ and $\{0001\}$ facets and solution, respectively, and linear sizes $L_{1}$ and $L_{2}$ are defined in Eq. (1). The data extracted for linear sizes of particle No. 1 from images given in Figs. $1 \mathrm{~b}$ to $\mathrm{f}$ yield for the average value of the aspect ratio an estimate $r_{L}=L_{1} / L_{2} \approx 2.7$, which in compliance with Eq. (8) predicts rather strong anisotropy for the surface energy of the $\mathrm{CH}$. Besides, the estimate $L_{1} / L_{2} \approx 2.7$ indicates that the growth rates of $\{10 \overline{10}\}$ and $\{0001\}$ facets also substantially differ. In turn, the difference in growth rates of different type of crystallographic facets mainly is caused by the two factors: anisotropy of the growth kinetic coefficient [9] and dependence of the supersaturation ratio at the surface of a facet on its crystallographic type [11]. According to Eq. (5), the latter factor may be associated with individual solubility of a facet of a certain crystallographic type, i.e. with anisotropy of the solubility, $C_{e}$. At supersaturation conditions, the growth process of a particle in solution results from the two competitive processes, crystallization and dissolution, with domination of the crystallization. Even at supersaturation conditions, detachment of a certain amount of adsorbed molecules (i.e, dissolution) takes place owing to local thermodynamic fluctuations of the supersaturation and temperature. At a given type of growth facet, the crystallization process (transition of solute molecules from solution into adsorptive layer and jump into crystallographic sites) is characterized by growth kinetic coefficient, whereas the dissolution process may be quantified through individual solubility of the facet [11], i.e. through local concentration of solute molecules in solution at the facet. Thus, in this approach, for a given facet the near-facet concentration of solute molecules is associated with solubility of this facet. However, in the case under consideration there is no evidence to argue that for a given facet the near-facet concentration of solute molecules equals to solubility of this facet. It is more reasonable to assume that the higher solubility of the facet the larger near-facet concentration of solute molecules in solution for this facet. Therefore, taking into account the above-presented estimate $\gamma_{1} / \gamma_{2} \approx 2.7$ (see Eq. (8)) for ratio of the surface energies of $\{10 \overline{10}\}$ and $\{0001\}$ facets, in our further analysis it is assumed that, at these facets, concentrations of solute molecules in solution differ. Accordingly, after replacements

$$
\beta \rightarrow \beta_{i}, \quad C_{e} \rightarrow C_{s i}, \quad d \rightarrow L_{i}, \quad(i=1,2)
$$


Eq. (7) is modified into the following set of expressions:

$$
L_{i}(t)=\sqrt{\left(\frac{2 D}{\beta_{i}}+d_{n}\right)^{2}+8 \Omega\left(C-C_{s i}\right) D\left(t-t_{n}\right)}-\frac{2 D}{\beta_{i}}, \quad(i=1,2)
$$

where the subscript values $i=1$ and 2 correspond to $<10 \overline{10}>$ and $<0001>$ growth directions (i.e. to $\{10 \overline{10}\}$ and $\{0001\}$ facets), respectively; $L_{i}, \beta_{i}$, and $C_{s i}$ are the linear size of the particle (see Eq. (1)), kinetic coefficient and the concentration of solute molecules in solution at facets of the type $i(i=1,2)$, respectively, and $D$ is the diffusion coefficient of solute molecules. According to Eq. (9), at $t=t_{n}$,

$$
L_{1}\left(t_{n}\right)=L_{2}\left(t_{n}\right)=d_{n},
$$

where now $d_{n}$ is the initial value of the sizes $L_{1}$ and $L_{2}$ at the beginning of the post-nucleation period. Equation (10) is a boundary condition that connects Eqs. (2), (4), and (9) and expresses a continuous variation of the linear sizes of a growing particle at transition of the nucleation process into post-nucleation growth stage.

Equation (9) yields the growth rates in directions of interest, $V_{1}$ and $V_{2}$, as

$$
V_{i}(t)=\frac{d L_{i}}{d t}=\frac{4 \Omega\left(C-C_{s i}\right) D}{\sqrt{\left(\frac{2 D}{\beta_{i}}+d_{n}\right)^{2}+8 \Omega\left(C-C_{s i}\right) D\left(t-t_{n}\right)}}, \quad(i=1,2)
$$

where, again, the subscript values $i=1$ and $i=2$ correspond to growth rates in directions $<10 \overline{10}>$ and $<0001>$, respectively.

In our further analysis, we need numerical value of the nucleus-water interfacial energy, $\gamma$, introduced through Eq. (4). To the best of our knowledge, there is no literature data of this parameter for $\mathrm{CH}$ in aqueous solution. Brunauer et al [4] determined experimentally the total surface energy of $\mathrm{CH}$ to be $\gamma_{t}=1.18 \mathrm{~J} / \mathrm{m}^{2}$ that may substantially differ from interfacial energy, $\gamma$, as these parameters differ in terms of the physical definition. The interfacial energy may be estimated from the following relationship [12] :

$$
\gamma=\frac{q k T}{\Omega^{2 / 3}} \ln \left(\frac{1}{\Omega C_{e}}\right)
$$


where $q$ is the so-called shape parameter of a crystalline nucleus and accepts a value $q=0.514$ if the shape of the nucleus is spherical. For data $\Omega=5.488 \times 10^{-29} \mathrm{~m}^{3}, \quad C_{e}=7.83 \times 10^{24} \mathrm{~m}^{-3}$, $k=1.38066 \times 10^{-23} \mathrm{~J} / \mathrm{K}, T=298 \mathrm{~K}$, and $q=0.514$, a value $\gamma=0.114 \mathrm{~J} / \mathrm{m}^{2}$ is calculated from Eq. (12) for nucleus-water interfacial energy. Note that the obtained estimate $\gamma=0.114 \mathrm{~J} / \mathrm{m}^{2}$ for interfacial energy between $\mathrm{CH}$ and water by a factor of about 10 is smaller than the total surface energy of $\mathrm{CH}, \gamma_{t}=1.18 \mathrm{~J} / \mathrm{m}^{2}$, reported by Brunauer et al [4].

Figure 4 presents experimental data and corresponding theoretical curves for linear sizes $d$, $L_{1}$ and $L_{2}$ and for aspect ratio $r_{L}=L_{1} / L_{2}$ versus time for particle No. 1 (see Figs. 1b to f ). For this particle, the experimental values of linear sizes $L_{1}$, and $L_{2}$ have been obtained through direct measurements of these parameters from recorded images presented in Figs. $1 \mathrm{~b}$ to $\mathrm{f}$. According to obtained experimental data, during the observation time-interval $31 \mathrm{~min}<t<118 \mathrm{~min}$, the sizes $L_{1}$ and $L_{2}$ increased in the ranges $0.60 \mu m<L_{1}<1.38 \mu m$ and $0.21 \mu m<L_{2}<0.51 \mu m$, respectively. The aspect ratio, $r_{L}=L_{1} / L_{2}$, calculated from experimental values $L_{1}$ and $L_{2}$, was practically stable during observation time, $r_{L} \approx 2.7$. In Fig. 4 , the linear dependence $d(t)$ shows the increase of the nucleus size according to Eq. (2) at nucleation period, $t<t_{n}$, whereas the curves $L_{1}(t)$ and $L_{2}(t)$ represent the best fits to the experimental data at post-nucleation period, $t>t_{n}$, according to Eq. (9). The best fits $L_{1}(t)$ and $L_{2}(t)$ were achieved in the following way. First, applying the method of polynomials least-squares fitting, we found interpolation curves $L_{1}^{(i n)}(t)$ and $L_{2}^{(i n)}(t)$ for experimental $L_{1}$ and $L_{2}$ data, respectively. The intersection point of curves $L_{1}^{(i n)}(t)$ and $L_{2}^{(i n)}(t)$ yields duration of the nucleation period and critical diameter of nucleus, $t_{n} \approx 15 \mathrm{~min}$ and $d_{n} \approx 0.05 \mu m$ (see Fig. 4). Next, using the data $\Omega=5.488 \times 10^{-29} \mathrm{~m}^{3}, k=1.38066 \times 10^{-23} \mathrm{~J} / \mathrm{K}$, $T=298 \mathrm{~K}, \gamma=0.114 \mathrm{~J} / \mathrm{m}^{2}, C_{e}=7.83 \times 10^{24} \mathrm{~m}^{-3}$, and $K_{s p} \approx 8.79 \times 10^{-6} \mathrm{M}^{3}$, from the set of Eqs. (4) and (5) the value of the actual concentration of solute molecules is calculated to be $C=$ $8.22 \times 10^{24} \mathrm{~m}^{-3}$. Thus, for each dependence $L_{i}(t)(i=1,2)$ given by Eq. (9), the number of unknown parameters reduced to three, $\beta_{i}, D$, and $C_{s i}$. Finally, it was obtained that, according to Eq. (9), the best fits of dependences $L_{1}(t)$ and $L_{2}(t)$ to experimental data are achieved at values of unknown parameters $\quad \beta_{1}=6254 \mu \mathrm{m} / \mathrm{min}, \beta_{2}=5920 \mu \mathrm{m} / \mathrm{min}, \quad D=222 \mu \mathrm{m}^{2} / \mathrm{min}$, $C_{s 1}=7.970 \times 10^{24} \mathrm{~m}^{-3}$, and $C_{s 2}=8.181 \times 10^{24} \mathrm{~m}^{-3}$. It should be noted that curves $L_{i}^{(i n)}(t)$ and $L_{i}(t)(i=1,2)$ practically coincide, by this reason in Fig. 4 we present only the latter ones. 
From Eqs. (2) to (5), the following data have been calculated for the next group of unknown parameters: $K=10.18 \times 10^{-6} \mathrm{M}^{3}, S=1.158, V_{n}=0.003 \mu \mathrm{m} / \mathrm{min}$, and $\beta_{n}=78 \mu \mathrm{m} / \mathrm{min}$. Then, using Eq. (11), the average growth rates in directions $<10 \overline{10}>$ and $<0001>$ during the postnucleation period have been evaluated to be $\overline{V_{1}}=0.014 \mu \mathrm{m} / \mathrm{min}$ and $\overline{V_{2}}=0.005 \mu \mathrm{m} / \mathrm{min}$, respectively.

In spite of a large number of fitting parameters, $\beta_{1}, \beta_{2}, D, C_{s 1}$, and $C_{s 2}$, it was not complicated to achieve the best fits for curves $L_{1}(t)$ and $L_{2}(t)$ (according to Eq. (9)) to experimental data (see Fig. 4) since the fitting procedure was simplified in that the expression given by Eq. (9) exhibits considerably different parametric dependences on kinetic and diffusion coefficients. According to Eq. (9), at the beginning of the post-nucleation period, $t_{n} \approx 15 \mathrm{~min}<t$ $20 \mathrm{~min}$ (see Fig. 4), the size variation is controlled by the surface kinetic processes and, accordingly, the main fitting parameters are the kinetic coefficients $\beta_{1}$ and $\beta_{2}$. At a later stage, $t$ $20 \mathrm{~min}$, from Eq. (9) it follows that the growth process is driven predominantly by the diffusion and, accordingly, the main fitting parameter is the diffusion coefficient, $D$.

Interestingly, for an infinitely large observation time, Eq. (9) yields for the aspect ratio the following limit:

$$
r_{L}^{(s t)}=\operatorname{Lim}_{t \rightarrow \infty}\left[L_{1}(t) / L_{2}(t)\right]=\sqrt{\frac{C-C_{s 1}}{C-C_{s 2}}}
$$

At determined values $C=8.22 \times 10^{24} \mathrm{~m}^{-3}, C_{s 1}=7.970 \times 10^{24} \mathrm{~m}^{-3}$, and $C_{s 2}=8.181 \times 10^{24} \mathrm{~m}^{-3}$, Eq. (13) yields for our case a limit $r_{L}^{(s t)} \approx 2.5$. In turn, Eq. (13) leads to another important limit

$$
\operatorname{Lim}_{C \rightarrow \infty} r_{L}^{(s t)}=1
$$

which predicts that at large concentrations of solute molecules the shape of a growing particle is expected to be geometrically more homogeneous in different crystallographic directions. From numerical analysis of Eq. (13), it follows that: (i) parameter $r_{L}^{(s t)}$ rapidly increases once the actual concentration of solute, $C$, approaches its near-surface concentration at $\{0001\}$ facets, $C_{s 2}=8.181 \times 10^{24} \mathrm{~m}^{-3}$; and (ii) a value $r_{L}^{(s t)}=1$ evaluated according to Eq. (14) is the lower limit of the aspect ratio.

Precipitating $\mathrm{CH}$ crystallites have been observed also in images recorded from another volumetric regions of the solution. For statistics, the values of the same parameters characterizing 
the growth process have been extracted in the same way (as above) from analysis of images of three another $\mathrm{CH}$ particles. For each physical parameter, the data extracted from analysis of images of four $\mathrm{CH}$ particles exhibit dispersion of no more than 5\% (for the sake of brevity, we do not present these additional images and corresponding data evaluated for growth parameters from these images). Therefore, all the physical parameters evaluated from analysis of the precipitation of particle No. 1 may be qualified as to be characteristic for the growth process of the $\mathrm{CH}$ phase in solution under investigation. According to plots presented in Fig. 4 for particle No. 1, the growth process of the $\mathrm{CH}$ crystals in solution may be quantitatively described as follows: At nucleation period with a duration of $t_{n} \approx 15 \mathrm{~min}$, the $\mathrm{CH}$ quasi-spherical nuclei grow in size at growth rates of about of $V_{n}=0.003 \mu \mathrm{m} / \mathrm{min}$. Only a small amount of these nuclei achieve at the end of this nucleation period the critical size $d_{n} \approx 0.05 \mu \mathrm{m}$ and continue to increase in size during the postnucleation period, $t>t_{n}$. At an initial stage of the post-nucleation period, $15 \min <t<25 \mathrm{~min}$, the aspect ratio of the particles rapidly increases from $r_{L}\left(t_{n}\right)=1$ to a maximal value $r_{L}^{(\max )} \approx 2.7$, which practically is maintained during the succeeding time-interval of the observation time, $25 \mathrm{~min}<t<118 \mathrm{~min}$. Such behavior of the aspect ratio is presumably caused by a rapid transformation of quasi-spherical shape of the nuclei into a polyhedral crystalline shape, and further transformation of the polyhedral shape into a stable hexagonal-prism shape (see Fig. 2). The former shape transformation may be associated with formation, except of $\{10 \overline{10}\}$ and $\{0001\}$ facets of the $\mathrm{CH}$, also of other type small-index crystallographic facets such as $\{1 \overline{2} 10\},\{10 \overline{1} 1\}$, and $\{10 \overline{1} 2\}$. The latter shape transformation results from gradual vanishing of all the type crystallographic facets except of the $\{10 \overline{10}\}$ and $\{0001\}$ ones. The $\mathrm{CH}$ crystals grow at post-nucleation period, $15 \mathrm{~min}<t<118 \mathrm{~min}$, through their preferential $\{1010\}$ and $\{0001\}$ facets with the average growth rates $\overline{V_{1}}=0.014 \mu \mathrm{m} / \mathrm{min}$ and $\overline{V_{2}}=0.005 \mu \mathrm{m} / \mathrm{min}$, respectively. Note that during the growth process, it is presumed that facets like $\{1 \overline{210}\},\{10 \overline{1} 1\}$, and $\{10 \overline{1} 2\}$ form and then vanish since their surface energies and, hence, the growth rates exceed the same parameters associated with $\{10 \overline{10}\}$ and $\{0001\}$ facets [13]. As it is predicted by obtained theoretical dependence $r_{L}(t)$ for the post-observation time $t>118 \mathrm{~min}$ [see Fig. 4 and Eq. (13)], the aspect ratio tends to a stationary value $r_{L}^{(s t)} \approx 2.5$ provided that evaluated values of concentrations $C, C_{s 1}$, and $C_{s 2}$ are maintained constant in time. Future analysis [14] will examine whether concentrations $C_{s 1}$ and $C_{s 2}$ introduced in Eq. (9) may be associated with dissolution rates and individual solubility of $\{10 \overline{10}\}$ and $\{0001\}$ facets, respectively. 
From Eqs. (13) and (14), it may be assumed that through manipulation of the actual concentration of dissolved stoichiometric molecules, $C$, it is possible to control the aspect ratio of precipitating particles of $\mathrm{CH}$ crystals. This has implications from the technological standpoint in the production of cementitious materials, because the aspect ratio of a crystal with known shape and type of crystallographic facets allows, in particular, quantitative estimates of a cement's adhesive ability at different environmental and physical conditions. A lower limit $r_{L}^{(s t)}=1$ evaluated in this work for the aspect ratio of precipitating $\mathrm{CH}$ crystals in aqueous solution is in agreement with experimental observations for this parameter by other authors [4-7] (see the relationship given by Eq. (1)).

This study demonstrated that the high resolution soft X-ray transmission microscopy may serve as an effective tool not only for observation of the hydration of cementitious particles in aqueous solutions [2], but is also a valuable technique for quantitative analysis of the throughsolution precipitation process of different stoichiometric phases of the cement paste. Experimental results on the basis of the proposed theoretical model enabled evaluation of the realistic data for the main physical parameters that characterize the growth process of the $\mathrm{CH}$ crystals: supersaturation ratio, kinetic and diffusion coefficients, nucleation critical size, crystal aspect ratio, and growth rates for different crystallographic facets. A theoretical justification is presented for the lower limit that exhibits the aspect ratio of the $\mathrm{CH}$ crystals in cementitious solutions and hardened cement pastes.

\section{Acknowledgments}

The research described in this publication was made possible in part by Award No. ARP2-2610YE-04 of the U.S. Civilian Research and Development Foundation for the Independent States of the Former Soviet Union (CRDF). The soft X-ray microscope at the ALS is supported by the Director, Office of Science, Office of Basic Energy Sciences, Materials Sciences and Engineering Division, of the U.S. Department of Energy under Contract No. DE-AC02-05-CH11231.

\section{References}

1. E.M. Gartner, K.E. Kurtis, P.J.M. Monteiro, Cem. Concr. Res. 30, 817 (2000).

2. M.C.G. Juenger, V.H.R. Lamour, P.J.M. Monteiro, E.M. Gartner, G.P. Denbeaux, J. Mater. Sci. Lett. 22, 1335 (2003).

3. K.E. Kurtis, P.J.M. Monteiro, J.T. Brown, W. Meyer-Ilse , Cem. Concr. Res. 28, 411 (1998).

4. S. Brunauer, D.L. Kantro, and C.H. Weise, Canad. J. of Chemistry 34, 729 (1956).

5. C. Rodriguez-Navarro, E. Hansen, and W.S. Ginell, J. Amer. Cer. Society 81, 3032 (1998).

6. J.P. Boyer and R.L. Berger, J. Amer. Cer. Society 63, 575 (1980).

7. H. Teramoto and S. Koie, J. Amer. Cer. Society 59, 522 (1976). 
8. P.K. Mehta, Cem. Concr. Res. 3, 1 (1973).

9. B.K. Vainshtain et al., Modern Crystallography V. 3, Formation of Crystals, Nauka, Moskow

1980.

10. R.L. Parker, Crystal Growth Mechanisms: Energetics, Kinetics and Transport, Solid State Physics,

V. 25, Academic Press, New York and London 1970.

11. E.V. Khamskii, Crystallization in Solutions, Nauka, Leningrad 1967, p. 49.

12. D. Kashchiev and G.M. van Rosmalen, Cryst. Res. Technol. 38, No. 7-8, 555 (2003).

13 R.A. Laudise, The Growth of Single Crystals, Prentice-Hall, New Jersy, 1970.

14. V.S. Harutyunyan, A.P. Kirchheim, P.J.M. Monteiro, A.P. Aivazyan, P. Fischer, J. Amer. Cer.

Society, to be published.

\section{Captions}

Fig. 1. Soft X-ray images of aqueous cement solution (ASTM Type II portland cement and 6\% $\mathrm{C}_{4} \mathrm{~A}_{3} \overline{\mathrm{S}}$ admixture; solution/solutes ratio was $10 \mathrm{~cm}^{3} / \mathrm{g}$ ) saturated with respect to $\mathrm{CH}$ and gypsum. Images were recorded at a time-interval $15 \mathrm{~min}$ to $118 \mathrm{~min}$ immediately after solution preparation from a fixed volumetric region. Scale bar is $1 \mu \mathrm{m}$. White arrow in image $(a)$ indicates a hydrating cement grain or a cluster of grains. In images (b) to (f), solid arrows indicate the growth process of a $\mathrm{CH}$ crystal (numbered as particle No. 1), for which a quantitative analysis has been conducted in terms of growth parameters; dashed white arrows indicate a $\mathrm{CH}$ crystal (numbered as particle No. 2) with imaged $\{0001\}$ basal facet, and dashed black arrows indicate another three growing crystals also identified as $\mathrm{CH}$.

Fig. 2. (a): Hexagonal-prism shape of a $\mathrm{CH}$ crystallite with $\{0001\}$ basal and $\{10 \overline{10}\}$ lateral facets. $L_{2}$ is the linear size along $<0001>$ growth direction. (b): View at a $\{0001\}$ facet. $L_{1}$ is the linear size of the crystallite in $<10 \overline{10}>$ growth directions.

Fig. 3. Soft X-ray microscopy image of aqueous cement solution (100\% $\mathrm{C}_{4} \mathrm{~A}_{3} \overline{\mathrm{S}}$ admixture; solution/admixture ratio was $10 \mathrm{~cm}^{3} / \mathrm{g}$ ) saturated with respect to $\mathrm{CH}$ and gypsum. Scale bar is $1 \mu \mathrm{m}$.

Fig. 4. Variation of the linear sizes $d, L_{1}$ and $L_{2}$ of particle 1 depending on time. At nucleation period, $0<t<t_{n}$, the particle size $d$ increases linearly according to Eq. (2) (solid inclined line). The horizontal solid line corresponds to nucleation critical diameter $d_{n}=0.05 \mu \mathrm{m}$. At post- 
nucleation period, $t>t_{n}$, the medium and small size dots and curves $L_{1}(t)$ and $L_{2}(t)$ represent experimental data and the best fits according to Eq. (9) for sizes $L_{1}$ and $L_{2}$, respectively; large dots and curve $r_{L}(t)=L_{1}(t) / L_{2}(t)$ represent experimental data and the theoretical curve of the aspect ratio over period $t>0$. In compliance with

boundary condition (10), $d\left(t_{n}\right) \equiv d_{n}=L_{1}\left(t_{n}\right)=L_{2}\left(t_{n}\right)$. At $t>25 \mathrm{~min}$, the aspect ratio is maintained at a maximal level $r_{L} \approx 2.7$. The horizontal dashed line corresponds to asymptotic limit $r_{L}^{(s t)}=2.5$ at $t=\infty$ according to Eq. (13).

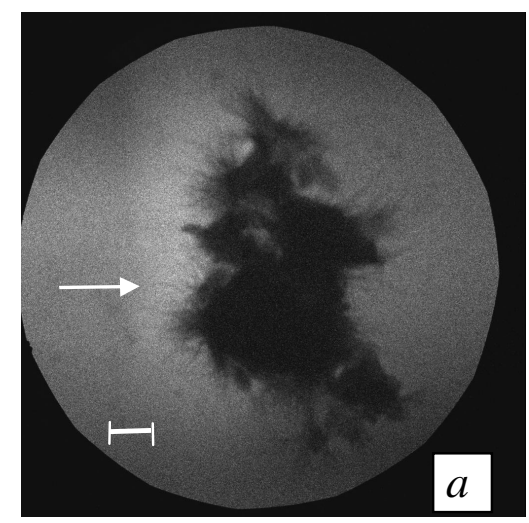

$15 \min$

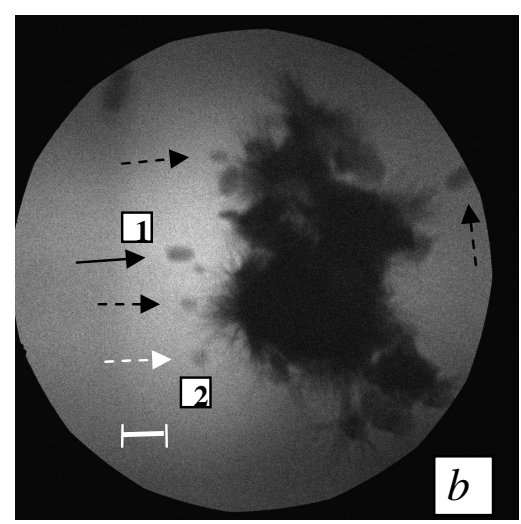

$31 \min$

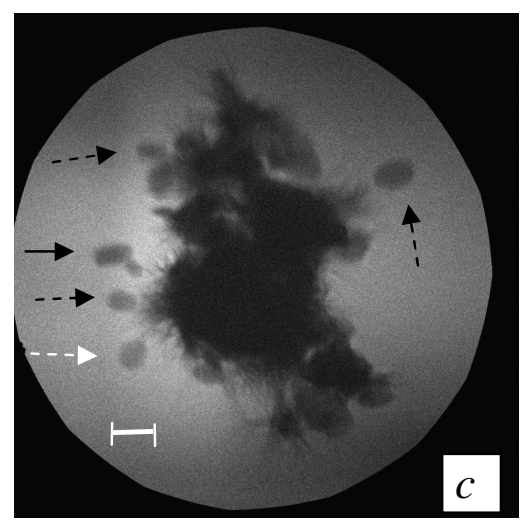

$44 \min$ 

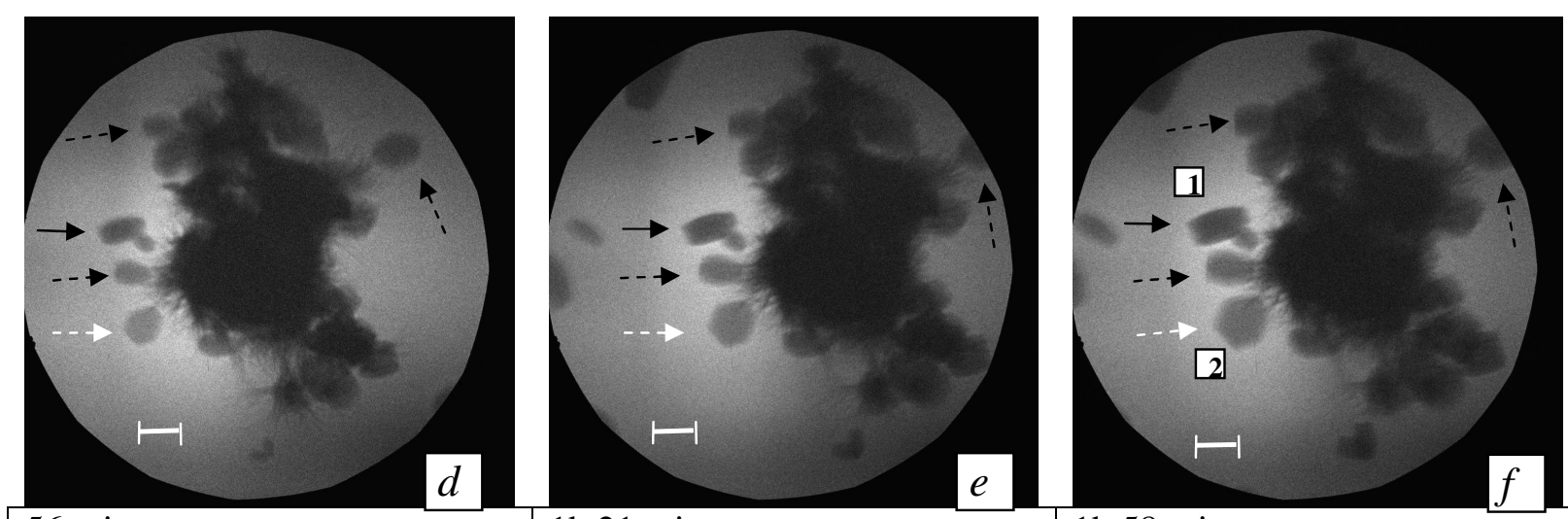

1h $58 \mathrm{~min}$

Fig. 1
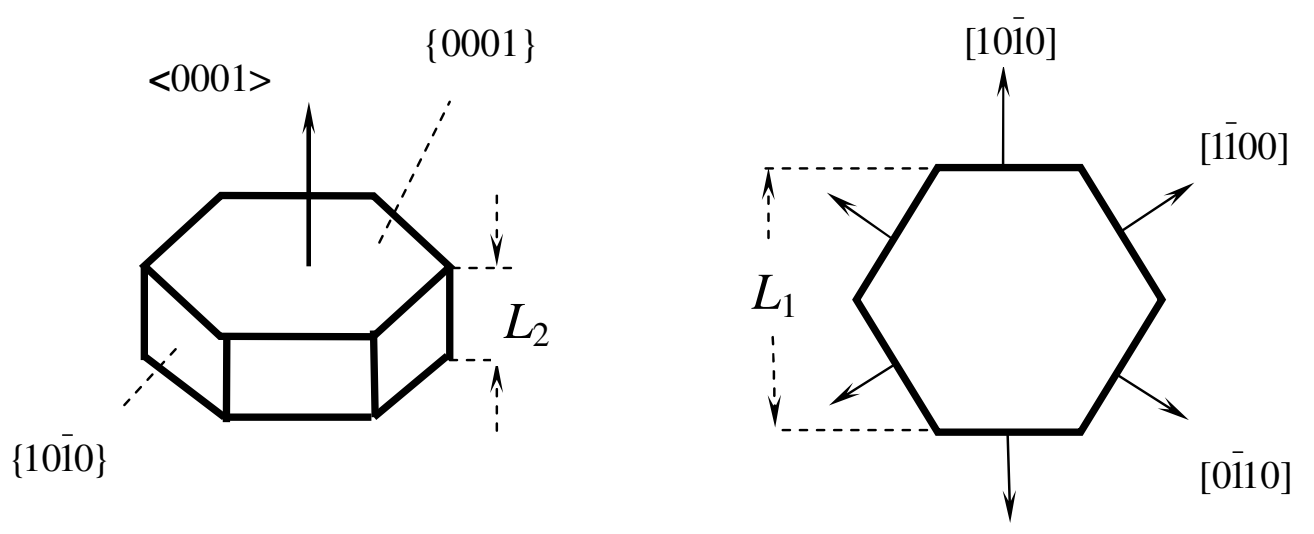
Fig. 2 


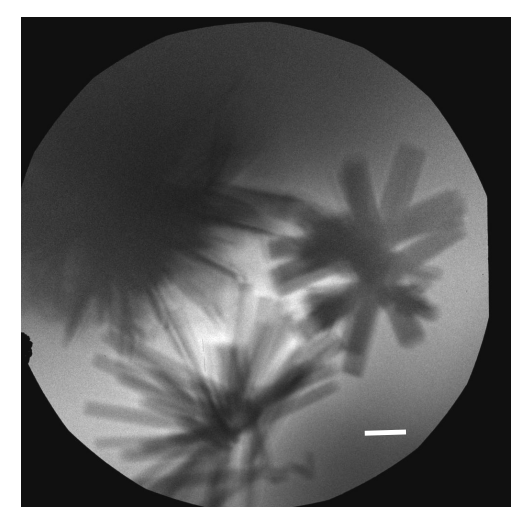

Fig. 3

囦 


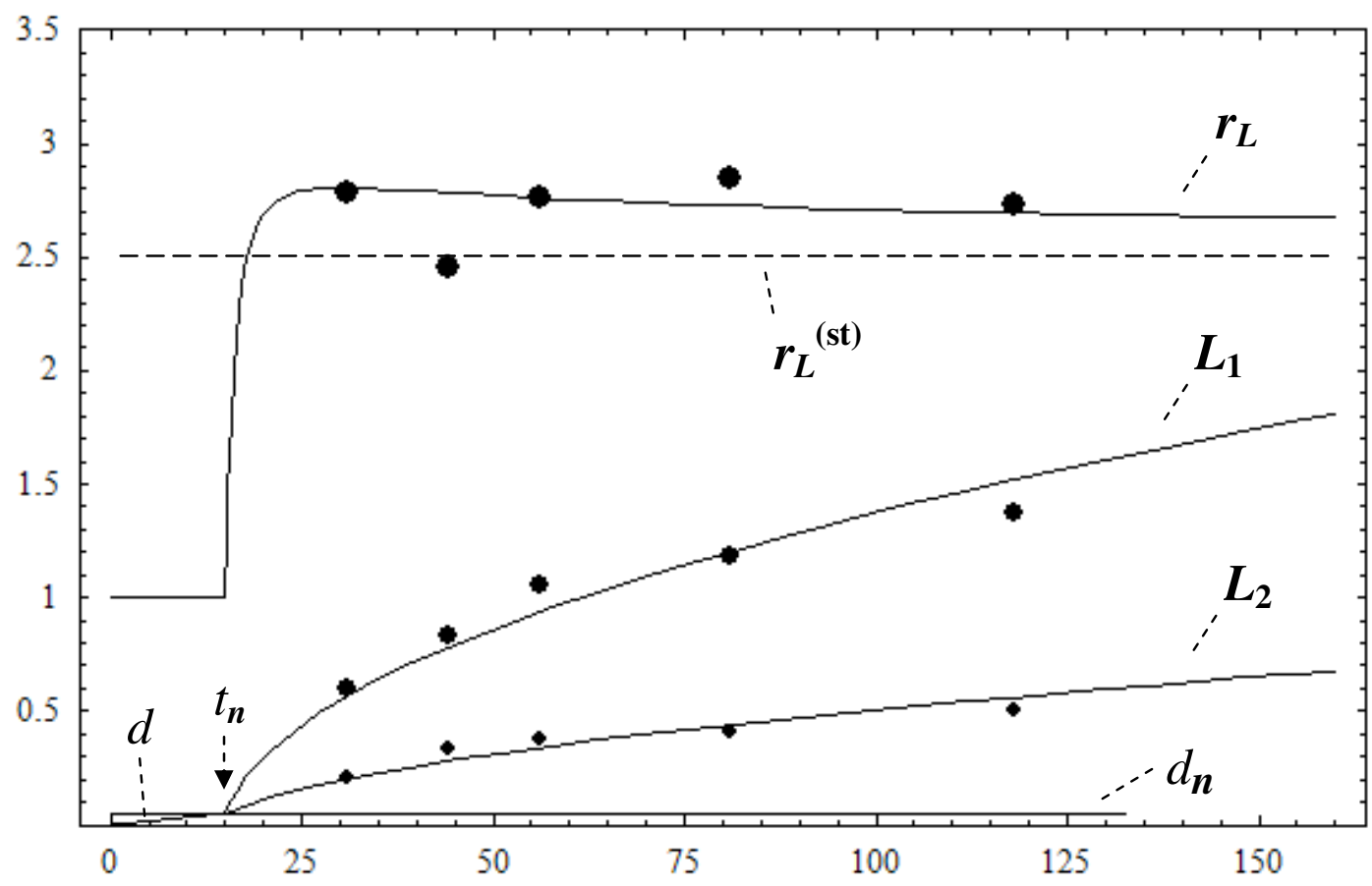

Time, $t$ (min.)

Fig. 4 